J. Nonlinear Var. Anal. 2 (2018), No. 1, pp. 91-102

Available online at http://jnva.biemdas.com

https://doi.org/10.23952/jnva.2.2018.1.07

\title{
INTERSECTION PROPERTIES, HYPERCONVEXITY AND JUNG CONSTANTS
}

\author{
PIER LUIGI PAPINI
}

Via Martucci, 19, 40136 Bologna, Italy

\begin{abstract}
Different kinds of intersection properties have been investigated. They are used to estimate projection constants, to define some classes of spaces (like hyperconvex spaces), and to study the Jung constants. In this paper, we study the relations among them and with some other properties, and also show that some notions and some results have been defined and studied again after some time of partial oblivion. Moreover, we raise several questions: probably some of them are highly non trivial.

Keywords. Intersection properties; Radius; Jung constant; hyperconvexity.
\end{abstract}

2010 Mathematics Subject Classification. 47A20, 41A28.

\section{INTRODUCTION}

Throughout the paper, $X$ denotes (unless otherwise stated) an infinite dimensional Banach space over the real field $R$. For $x \in X$ and $r \geq 0$, we denote by $B(x, r)$ the ball centered at $x$ of radius $r$. Given a bounded nonempty set $A \subset X$, let

$$
\delta(A)=\sup \{\|a-b\|: a, b \in A\}, \quad(\text { diameter of } A) .
$$

For $x \in X$,

$$
\begin{gathered}
r(A, x)=\sup \{\|a-x\|: a \in A\}, \quad(\text { radius of } A \text { from } x), \\
r(A)=\inf \{r(A, x): x \in X\}, \quad(\text { radius of } A) .
\end{gathered}
$$

Concerning the last definition: any point (if there exists) realizing the minimum is called a center of $A$. The conditions: bounded closed and convex will be abbreviated as $b c c$.

Consider the following constants:

$$
\begin{aligned}
J(X) & :=\sup \left\{\frac{2 r(A)}{\delta(A)}: A \text { bounded, } \delta(A)>0\right\} \text { (Jung constant), } \\
J_{f}(X): & =\sup \left\{\frac{2 r(A)}{\delta(A)}: A \text { finite, } \delta(A)>0\right\} \text { (finite Jung constant). }
\end{aligned}
$$

We recall the following simple estimates (see for example [2]). For any $X$, we have

$$
1 \leq J_{f}(X) \leq J(X) \leq 2 .
$$

In this paper, we deal essentially with intersections of balls. A basic reference for this subject is still [26], where several problems were solved, while some others gave rise to other studies in the area. There are important connections with other topics, like projections, the dual space, the extension of operators.

E-mail address: pierluigi.papini@ unibo.it.

Received December 29, 2017; Accepted January 11, 2018.

(C) 2018 Journal of Nonlinear and Variational Analysis 
But here we shall try to study intersection properties "in themselves", mainly avoiding to consider these connections.

Though the notions we consider have mostly been introduced more than half century ago, some questions concerning them remain unanswered. The organization of this paper is the following. In Section 2, we consider intersection properties. In Section 3, we discuss hyperconvexity and some related notions. Some partly new properties are considered in Section 4. In Section 5, we deal with the finite Jung constant. Several problems are collected in the Section 6. Finally, in Section 7, we indicate two examples and we partially summarize the situation with a scheme.

\section{INTERSECTION PROPERTIES}

Consider a family $\mathscr{F}$ of balls:

$$
\mathscr{F}=\left\{B\left(x_{\alpha}, r_{\alpha}\right): \alpha \in \mathscr{A}\right\},
$$

where $\mathscr{A}$ is a family of indexes. We say that they are pairwise intersecting if for any two indexes $\alpha, \beta \in \mathscr{A}$, we have $r_{\alpha}+r_{\beta} \geq\left\|x_{\alpha}-x_{\beta}\right\|$. We can consider several intersection properties, which appear frequently in the literature (for most of them one can see [26, p.30], or [24, p.207]):

$$
I P_{2, n}(n \in N) ; I P_{2, f} ; I P_{2, \infty} .
$$

They correspond to the fact that for a family of pairwise intersecting balls, respectively, any $n$ intersect; any finite number of them intersect ( $f$ stands for finite), they all intersect.

If we consider the analogous property, but with the restriction that all balls in the family have the same radius, we shall indicate the corresponding (weaker) properties by:

$$
R I P_{2, n}(n \in N) ; R I P_{2, f} ; R I P_{2, \infty} .
$$

It is clear the meaning that $I P_{f, \infty}$ and $R I P_{f, \infty}$ will have: in Section 4 we shall consider these two properties, that we shall simply indicate by FIP and RFIP.

When a space lacks a property, it can be interesting to estimate "how far" it is from satisfying it. So we are led to define several parameters. According to [22], the constants $E(X), E_{f}(X)$ we are defining will be said to be exact if the inf defining them is also a minimum; the same definition of exactness will apply to $J(X), J_{f}(X)$. Starting from $I P_{2, \infty}$, we shall consider the following number, studied by Grünbaum (in [21] for finite dimensional spaces; in [22] in general). Set: $\operatorname{Exp}(X)=\{\lambda \geq 1$ : given a family $\left\{B\left(x_{\alpha}, r_{\alpha}\right): \alpha \in \mathscr{A}\right\}$ of pairwise intersecting balls, we have $\left.\bigcap_{\alpha \in \mathscr{A}} B\left(x_{\alpha}, \lambda r_{\alpha}\right) \neq \emptyset\right\}$, where $\mathscr{A}$ is a family of indexes. Define

$$
E(X):=\inf \{\operatorname{Exp}(X)\} \text { (expansion constant). }
$$

The following facts were indicated in [22, pp.198-200]: $J(X) \leq E(X) \leq 2$ always. The constant $E(X)$ has the same value as other two constants considered there (called retraction constant, projection constant); moreover an example of a space $X$ where $J(X)=2$ is exact while $E(X)$ is not exact was given.

A space where $E(X)=\lambda(\lambda \geq 1)$ and it is exact (so $\left.\bigcap_{\alpha \in \mathscr{A}} B\left(x_{\alpha}, \lambda r_{\alpha}\right) \neq \emptyset\right)$, is called an $E_{\lambda}$ space; see $[26, p .82]$ and the references therein.

Remark 2.1. $E_{\lambda}$ spaces were called in $[15, \mathrm{p}$.461] $\lambda$-hyperconvex; but the last term was in general used to indicate a different notion (see [23]). Indeed, different notions of (ball) intersections, by using intersections of balls with subspaces (called $n$-intersection properties), have been considered (see, for 
example, [25, Section 6]): they are useful, for example, in the context of best approximation theory. In turn, $\lambda$-hyperconvexity according to [23] generalizes one of them. We shall not consider these notions here.

If a space $X$ has $E(X)=1$, and this is exact, we say that $X$ is hyperconvex (this notion has been considered for metric spaces in general). For Banach spaces, it is equivalent to the fact that $X$ is a $\mathscr{P}_{1}$ space (i.e., a space $C(K), K$ a compact Hausdorff extremally disconnected set). Several equivalent conditions for these spaces, which are also called 1-injective, are known: see, for example, [26, pp.5253].

So $E(X)$ is a way to measure the "distance" for a space from being hyperconvex. If we use $I P_{2, f}$ (instead of $\left.I P_{2, \infty}\right)$, we can define analogously a constant

$E_{f}(X):=\inf \left\{\lambda \geq 1\right.$ : given a finite family $\left\{B\left(x_{\alpha}, r_{\alpha}\right): \alpha \in \mathscr{A}_{f}\right\}$ of pairwise intersecting balls, we have $\left.\bigcap_{\alpha \in \mathscr{A}_{f}} B\left(x_{\alpha}, \lambda r_{\alpha}\right) \neq \emptyset\right\} \quad\left(\mathscr{A}_{f}\right.$ a finite family of indexes).

If $X$ satisfies $I P_{2, f}$, then we shall say that it is finitely-hyperconvex (see [23, p.22]): we prefer this term instead of the generally used $\aleph_{0}$-hyperconvex (see [3, p.410], [29, pp.201-202], [15, p.457] or [4, p.136]). It is simple to prove that starting from $R I P_{2, \infty}$ or, respectively, from $R I P_{2, f}$, we have the following equalities:

$J(X)=\inf \left\{\lambda \in R\right.$ : given a family $\left\{B\left(x_{\alpha}, r\right)\right\}$ of pairwise intersecting balls (with the same radius), we have $\left.\bigcap_{\alpha \in \mathscr{A}} B\left(x_{\alpha}, \lambda r\right) \neq \emptyset\right\} \quad(\mathscr{A}$ a family of indexes).

$J_{f}(X)=\inf \left\{\lambda \in R\right.$ : given a finite family $\left\{B\left(x_{\alpha}, r\right): \alpha \in \mathscr{A}_{f}\right\}$ of pairwise intersecting balls (with the same radius), we have $\left.\bigcap_{\alpha \in \mathscr{A}_{f}} B\left(x_{\alpha}, \lambda r\right) \neq \emptyset\right\}$

$\left(\mathscr{A}_{f}\right.$ a finite family of indexes).

It is not difficult to see that the above constants are "exact", according to the language of [22], exactly when all sets $A$ realizing $J(X)(2 r(A) / \delta(A)=J(X))$, or $J_{f}(X)$, have a center, According to these definitions these constants are always exact when their value is 2 . We prefer to use here a stronger definition of exactness.

A space $X$ is said to admit centers if every bounded (equivalently: every bcc) subset of $X$ has (at least) a center. In this case we say that $J(X)$ is $E X A C T$. Similarly, we say that $J_{f}(X)$ is EXACT if all finite sets have centers. The two definitions of exactness coincide if $J(X)=1$. An example showing that in general the last definition is strictly stronger, will be given in Section 7 (Example 7.1).

In many classical Banach spaces, all bcc sets have a center: examples of sets without center are given, in general, in rather artificial spaces (see, for example, [32]). With regard to this, in Section 6 we indicate a couple of open questions.

Remark 2.2. The "self Jung constant" is different from the Jung constant (see, for example, [2]). We shall not consider it here, though, it may also be more interesting for applications (mainly in the context of fixed point theory).

We collect in a proposition some results, contained in [26], that we shall use. We say that a property like $I P_{2, f}$, is almost satisfied if: given any finite set of pairwise intersecting balls $B\left(x_{\alpha}, r_{\alpha}\right)$, we have

$$
\bigcap_{\alpha} B\left(x_{\alpha}, r_{\alpha}+\varepsilon\right) \neq \emptyset, \quad \forall \varepsilon>0 .
$$


Proposition 2.1. (1) ([26, Theorem 4.1 and the Remark to it]) $I P_{2,4}$ implies $I P_{2, f}$ (but $I P_{2,3}$ does not suffice, see [26, p.32 and p.59]). Another proof of this result was given in [25, Theorem 2.12].

(2) ([26, Lemma 4.2]) Assume that $X$ almost satisfies $I P_{2, n}$ (for some $n \in N$ ). Then it has the $I P_{2, n}$. Therefore $I P_{2, f} \Leftrightarrow$ almost $I P_{2, f}$. Moreover (see [3, Section 3, Theorem 4]): the same is true when instead of $n$ we have $\infty$, or countable.

(3) ([26, Theorem 4.3]) For all $n \geq 3, R I P_{2, n}$ implies $I P_{2, n}$. Thus $R I P_{2, f}$ implies $I P_{2, f}\left(J_{f}(X)=\right.$ $1+$ exact is the same as $E_{f}(X)=1+$ exact; see Theorem 5.1 in Section 5 below).

As a consequence of (2) of Proposition 2.1, we have

$$
\text { if } E_{f}(X)=1 \text {, then it is exact; if } J_{f}(X)=1 \text {, then it is exact. }
$$

Theorem 3.1 below generalizes (3) of Proposition 2.1. We recall that also [25] contains some related results, mainly concerning $I P_{2,3}, I P_{2,4}$.

\section{HYPERCONVEXITY AND RELATED NOTIONS}

In the previous section we have defined hyperconvex spaces. Now we recall a well known result (see $[13,26])$ : in particular, it indicates that $I P_{2, \infty}$ and $R I P_{2, \infty}$ are equivalent. For a result of this type, see [5, Theorem 4.6].

Theorem 3.1. The following conditions are equivalent

(1) $X$ is hyperconvex $(E(X)=1$ and is exact);

(2) $E(X)=1$;

(3) $J(X)=1$ and is exact;

(4) $J(X)=1($ or: $\delta(A)=2 r(A)$ for every bcc set $A)$.

Therefore, $E(X)=1$ (also: $J(X)=1$ ) implies its exactness (see [26, Theorem 6.10]); we can say that "almost" hyperconvexity implies hyperconvexity. So these conditions imply that $X$ admits centers.

Note that the strongest implications in Theorem 3.1: (4) $\Rightarrow(3) \Rightarrow(1)$, were proved in [13]. Another proof, based on the implication (4) $\Rightarrow(2)$, was given in [17, Theorem 4]; see also [18] for another proof and some improvements.

Remark 3.1. A space satisfying (3) ( $\equiv R I P_{2, \infty}$, balls all with the same radius) was called in [29, pp.201202] weakly hyperconvex; at that time [13] had not yet appeared.

We add a characterization of hyperconvexity. We recall that a bounded set $A$ with $\delta(A)>0$ is said to be diametrically maximal, (DM) for short, if the addition of any point to the set increases the diameter. We have the result which follows; since paper [17] is not easily available, we indicate a simple way to obtain such result.

Theorem 3.2. (see [17, Theorem 2]). A space $X$ is hyperconvex if and only if the following is true: a bounded set $A$ is (DM) if and only if it is a ball.

Proof. As proved in [8, Theorem 4.1], we have

$$
J(X)=\sup \left\{\frac{2 r(A)}{\delta(A)}: A \text { is }(D M)\right\} .
$$


So it is clear that from the condition (DM) $\Rightarrow$ ball, we obtain $J(X)=1$. Conversely, let $J(X)=1$; thus, in particular, all (DM) sets satisfy $2 r(A)=\delta(A)$. But this equality, for (DM) sets, implies that they are balls (see [27, Proposition 4.3]).

Countable properties. We indicate some generalizations of our properties: instead of requiring an intersection property for all families of balls, we ask it for countable families. The constants we obtain are the same considered before in case a space is separable, but not in general.

As far as we know, apart from [10], next parameter and its relation with $J(X)$ have not been considered in any paper. We say that $A$ is separable if it is the closure of a countable set. Set

$$
J_{0}(X):=\sup \left\{\frac{2 r(A)}{\delta(A)}: A \text { separable, } \delta(A)>0\right\} \text { (separable Jung constant); }
$$

$\left(J_{f}(X) \leq J_{0}(X) \leq J(X)\right.$ always). Clearly, $J(X)=J_{0}(X)$ if $X$ is separable; this is not true in general: the example in [23, Theorem 5] shows this.

So a "countable" weakening of $I P_{2, \infty}$ leads to a new class of spaces. Suppose that the property $I P_{2, \ldots .}$ is satisfied for countable many balls (instead of infinite, or finitely many: see [23, p.22] and also [1, Lemma 4.3]). We speak of sequential hyperconvexity if every countable family of mutually intersecting balls has nonempty intersection; we shall indicate this property by $I P_{2,0}$.

The spaces satisfying $I P_{2,0}$ are called separably 1-injective spaces. So, for these spaces, $J_{0}(X)=$ $1+$ is exact. Recently there is a renewed interest in this class of spaces: see [4]. Indeed, they are often defined in an equivalent way, see [4, Proposition 2.30]. Sequential hyperconvexity does not imply hyperconvexity. So, we can define the constant $E_{0}(X)$ (instead of $E(X)$ ), that in general can be smaller if $X$ is not separable. An example where $E_{0}(X)=1$ (and is exact), while $E(X)=2$ (exact) is given in [23, Theorem 5]. For more examples concerning separably 1-injective spaces, one can refer to [4, p.26].

To obtain examples of spaces which are separably 1-injective but not 1-injective, according to the implications in (4.1) in Section 4 below, we must consider spaces not satisfying any of the properties implying (R) indicated there.

We do not know if the following fact is true (compare with Theorems 3.1 and 5.1).

Conjecture The following are equivalent:

(1) $X$ is separably 1 -injective (it satisfies $I P_{2,0}$ );

(2) $X$ almost satisfies $I P_{2,0}$;

(3) $J_{0}(X)=1+$ exact;

(4) $J_{0}(X)=1 \quad($ or: $\delta(A)=2 r(A)$ for every separable, bcc set $A)$.

It is clear that $(1) \Rightarrow(2) \Rightarrow(4) ;(1) \Rightarrow(3) \Rightarrow(4)$. Also, we have (according to the second part of Proposition 2.1, (2)): (1) $\Leftrightarrow(2)$. Therefore (1) $\Leftrightarrow(2) \Rightarrow(3) \Rightarrow$ (4).

\section{SOME OTHER DEFINITIONS; THE FINITE INTERSECTION PROPERTY}

In this section, we deal mainly with (restricted) finite intersection property. It is not surprising that these properties can greatly influence the behavior of the constants that we have introduced before.

Consider the following property, which is also based on the intersection properties: (R) $J(X)=J_{f}(X)$.

Equality $(\mathrm{R})$ is true in all dual spaces (see [2, 2.1 Proposition (a)]). Now we shall discuss some related facts. 
Recall the following property: its definition goes back to Grünbaum, see [22, Definition 3] (it was also used in [12]), then considered in [24, p.207], [26, p.59]. See also [5], [16], [19], [20], whose authors did not refer to the previous papers.

FIP (finite intersection property, $\equiv I P_{f, \infty}$ ). Consider a family $\mathscr{F}$ of balls in $X$. In case every finite subfamily of $\mathscr{F}$ intersects, then the intersection of the whole family is nonempty. A slightly weaker property is the following (see [26, p.30], [24, p.207], or [16, p.407]). Say that $X$ has the RFIP (restricted finite intersection property $\equiv R I P_{f, \infty}$ ) if given in $X$ a family $\mathscr{F}$ of balls with the same radius, if every finite subfamily of $\mathscr{F}$ intersects, then the intersection of the whole family is nonempty. Also, consider the following property:

$\left(\mathrm{R}^{+}\right)$given any bounded set $A$ in $X$, we have $r(A)=\sup \left\{r\left(A_{f}\right): A_{f}\right.$ is a finite subset of $\left.A\right\}$.

The following implications hold for any space:

$$
\text { reflexivity } \Rightarrow \text { being a dual } \Rightarrow F I P \Rightarrow R F I P \Rightarrow\left(R^{+}\right) \Rightarrow(R) \text {. }
$$

The first, the second, the third and the fifth implication are well known or trivial. Also the fourth one is easy, but we shall sketch below a proof of it (Proposition 4.1).

Concerning the second implication: the reverse is not true; for example, FIP also holds in the spaces $L_{1}(\mu)$ (for some measure $\mu$ : see [26, p.60]) and of course in $\mathscr{P}_{1}$ spaces. Conditions implying the reverse implications in (4.1), mainly for the first and the second of them, were discussed in several papers; see, for example, [19, Corollary 6], [20], [11, Theorem 3.1]; the questions concerning the equivalence between the second and the third condition had been already raised in [22, p.199]. For the (possible) implication RFIP $\Rightarrow$ FIP, see [16] and the references therein.

Note that $c_{0}$ does not satisfy (R), so it does not satisfy the others properties in (4.1). For example, the family of balls of radius $1 / 2$, centered at the points of the natural basis, has empty intersection: so RFIP is not satisfied.

Concerning RFIP: it is clear that if every finite subfamily intersects and the radius of all balls is $\rho$, then the union of all balls in the family has diameter not larger than $4 \rho$. Therefore RFIP says something only for families of balls such that $\bigcup_{a \in \mathscr{A}} B\left(x_{a}, r_{a}\right)$ is bounded.

So we can also indicate a property which is between FIP and RFIP:

$F I P^{-}$Consider a family $\mathscr{F}=\left\{B\left(x_{a}, r_{a}\right): a \in \mathscr{A}\right\}$ of balls in $X$. Assume that $\bigcup_{a \in \mathscr{A}} B\left(x_{a}, r_{a}\right)$ is bounded. If every finite subfamily of balls intersects, then the intersection of the whole family is nonempty.

Note that the assumption we added means the following:

$$
\text { both }\left\{x_{a}: a \in \mathscr{A}\right\} \text { and }\left\{r_{a}: a \in \mathscr{A}\right\} \text { are bounded. }
$$

So the assumption of only one of these two conditions would be formally weaker. But the condition $\left\{r_{a}: a \in \mathscr{A}\right\}$ is bounded, without the boundedness of $\left\{x_{a}: a \in \mathscr{A}\right\}$, makes impossible the nonemptiness of the intersection of finite families.

We do not know anything concerning the (possible) implications RFIP $\Rightarrow$ FIP $^{-}$and FIP $^{-} \Rightarrow$ FIP. Note that in many cases when FIP is used, the condition FIP ${ }^{-}$would suffice.

Proposition 4.1. The following implication is true

$$
R F I P \Rightarrow\left(R^{+}\right)
$$


Proof. Assume that $X$ has the RFIP. Consider a bounded set $A$ and let $r(A)=\rho$. Thus for any $\varepsilon>0$, we have $\bigcap_{a \in A} B(a, \rho-\varepsilon)=\emptyset$. This implies, according to $R F I P$, that for some finite set $A_{f}=\left\{a_{1}, \ldots, a_{n}\right\} \subset A$ we have

$$
\bigcap_{a \in A_{f}} B(a, \rho-\varepsilon)=\emptyset .
$$

Thus $r\left(A_{f}\right) \geq \rho-\varepsilon$. Since $\varepsilon>0$ is arbitrary, this implies $\left(R^{+}\right)$. This proves the proposition.

Next result shows that in some spaces the reverse implication holds.

Proposition 4.2. In a space $X$ which admits centers and satisfies $\left(R^{+}\right)$, also the RFIP holds.

Proof. The thesis is: let $\mathscr{F}=\{B(a, \rho): a \in A\}$ (for some $\rho>0$ ) be a family of balls in $X$ with $\bigcup_{a \in A} B(a, \rho)$ bounded. If any finite subset of them intersects, then the whole family intersects. We shall prove it by contradiction.

Assume that $\bigcap_{a \in A} B(a, \rho)=\emptyset$ (for some $\rho>0$ ). If $X$ admits centers, $r(A)>\rho$. According to $\left(R^{+}\right)$, this implies $r\left(A_{f}\right)>\rho$ for some finite subset $A_{f}$ of $A$. Therefore, $\bigcap_{a \in A_{f}} B(a, \rho)=\emptyset$. This shows that $X$ satisfies the RFIP.

Remark 4.1. It was proved in [31, Section 2], that there are spaces where all finite sets, but not all bounded sets, have centers. In such spaces, we can find a set $A$ of radius $\rho$ (without center), so that $\bigcap_{a \in A} B(a, \rho)=\emptyset$, while $\bigcap_{a \in A_{f}} B(a, \rho) \supset \bigcap_{a \in A_{f}} B\left(a, r\left(A_{f}\right)\right) \neq \emptyset$ for every finite subset $A_{f}$ of $A$. Thus in these spaces the RFIP does not hold. But probably in these spaces also (R) fails.

Note that exactness of $E(X)$ always holds if $X$ satisfies FIP; see [22, Theorem 3]. Indeed, it is not difficult to see that the FIP also implies exactness of $E_{f}(X)$ : but in fact, if the FIP holds, then we have (compare with (R) and (4.1)):

$$
E(X)=E_{f}(X) \text {. }
$$

More or less with the same proof, we can also obtain the following.

Proposition 4.3. If the FIP holds, then $J(X)=J_{f}(X)$ is EXACT.

Proof. The equality $J(X)=J_{f}(X)$ in this case is known (see (4.1)). Let $A$ be a bounded set. Set, for $\varepsilon>0$ and $a \in A: B_{a, \varepsilon}=B(a, r(A)+\varepsilon)$. Take a finite family of such balls, say $B_{a_{1}, \varepsilon_{1}}, \ldots, B_{a_{n}, \varepsilon_{n}}$. Let $\bar{\varepsilon}=\min \left\{\varepsilon_{i}: i=1, \ldots, n\right\}$. Then

$$
\bigcap_{i=1}^{n} B\left(a_{i}, r(A)+\varepsilon_{i}\right) \supset \bigcap_{i=1}^{n} B\left(a_{i}, r(A)+\bar{\varepsilon}\right) \supset \bigcap_{a \in A} B(a, r(A)+\bar{\varepsilon}) \neq \emptyset .
$$

Acording to the FIP,

$$
\bigcap_{a \in A}\left\{B_{a, \varepsilon}: \forall \varepsilon>0\right\}=\bigcap_{\varepsilon>0}\left(\bigcap\left\{B_{a, \varepsilon}: a \in A\right\}\right) \neq \emptyset .
$$

The last set is the set of centers of $A$, which is therefore nonempty.

Now, we define

$\Phi(X):=\inf \left\{\alpha \geq 1\right.$ : given a family of balls $\left\{B\left(x_{i}, r_{i}\right)_{i}\right\}$ such that any finite number of them intersect, then $\left.\bigcap_{i} B\left(x_{i}, \alpha r_{i}\right) \neq \emptyset\right\}$.

This notion is connected with the FIP (which corresponds to $\Phi(X)=1+$ exact). Clearly, $\Phi(X) \leq E(X)$. Finally, let 
$R \Phi(X):=\inf \left\{\alpha \geq 1\right.$ : given a family of balls $\left\{B\left(x_{i}, r\right)_{i}\right\}$ such that any finite of them intersect, then $\left.\bigcap_{i} B\left(x_{i}, \alpha r\right) \neq \emptyset\right\}$.

This notion is connected with $R I P_{f, \infty}$ (which corresponds to $R \Phi(X)=1+$ exact). Clearly, $R \Phi(X) \leq$ $\Phi(X)$. Also, $R \Phi(X) \leq J(X)$.

Some results concerning the equalities $J_{f}(X)=J_{0}(X)$ and $J_{0}(X)=J(X)$, similar to (4.1), could be given by weakening the conditions FIP and RFIP (in terms of countability).

We discuss briefly another akin condition, considered in a few papers. We define it through an equivalent condition (see [6, Proposition 2.9]), more connected with our discussion. We say that $X$ satisfies (GC) (generalized centers) if for all $n \in N$, if $n$ balls $B\left(x_{i}, r_{i}\right)$ almost intersect, then they intersect. In particular, when (GC) is satisfied, every finite set has a center; see also [5, Remark 2.5].

A space with FIP satisfies (GC) (see [6, p.207]), but the converse is not true: for example, a space $X$ with $J_{f}(X)=1$ (in particular: $c_{0}$ ) has (GC) (see [6, p.211]); $c_{0}$ fails all properties in (4.1). Moreover, (GC) is separably determined (i.e., if $Y$ is (GC) for every separable subspace $Y \subset X$, then also $X$ is (GC)): compare with [6, Proposition 4.1].

\section{Finite JUNG CONSTANT}

In this section, we indicate some relations among the conditions $J_{f}=1, \operatorname{or} R I P_{2, f}\left(J_{f}=1+\right.$ exact $)$, and $I P_{2, f}$. Clearly $E_{f}(X) \geq J_{f}(X)$ always.

It was proved in [9], with a non trivial proof, that $J_{f}(X)=1$ if and only if $X$ is a predual of $L^{1}(\mu)$. This fact was reproved in [28], where (Corollary 1 there) it was also proved that if $J_{f}(X)=1$, then this constant is exact. Practically [9] (and [10]), remained unknown: probably also because they used a slightly different language, not quoting Jung constant. Indeed, it was then proved in [14] that a careful reading of results of [26] brings to the same characterization of $J_{f}(X)=1$. Moreover, the following fact was indicated in [14, Theorem 2.7]: $J_{f}(X)=1$ if and only if every four-point set of diameter 1 has radius $1 / 2$ and has a center. According to (1) of Proposition 2.1 something similar (in terms of 4 balls) could probably be said for $E_{f}(X)$.

Other conditions equivalent, respectively, to $E_{f}(X)=1$, and to $J_{f}(X)=1$, are given in [26, Theorem 5.4] and, respectively, in [26, Theorem 6.1]. By using part of the last theorem, together with the results now indicated, we have the following result (analogous to Theorem 3.1, and practically contained in [26]).

Theorem 5.1. The following facts are equivalent

(1) $I P_{2, f}$ holds (or: $E_{f}(X)=1$ and is exact);

(2) $E_{f}(X)=1$;

(3) $I P_{2,4}$ holds;

(4) $J_{f}(X)=1$;

(5) $R I P_{2, f}$ holds (or: $J_{f}(X)=1$ and $X$ admits centers for finite sets);

(6) $X^{*}$ is an $L^{1}(\mu)$ space;

(7) $X^{* *}$ is a $\mathscr{P}_{1}$ space.

Remark 5.1. For another result of this type, see [5, Theorem 4.2]. In [30], another constant is considered: according to the main result there, condition 4 is equivalent to being equal to 1 such constant. Referring 
to the previous result, we like to recall the following example (see [7, Example 5.2]): there exists a three-point set $A$ without center, such that $2 r(A)=\delta(A)=1$.

Proposition 5.1. We always have $\frac{J(X)}{J_{f}(X)} \leq R \Phi(X)$. In particular, $R \Phi(X)=1$ implies $J(X)=J_{f}(X)$ $(\operatorname{property}(R))$.

Proof. Let $A$ be a bounded set with $\delta(A)=1$. For any finite subset $A_{f}$ of $A$, we have $2 r\left(A_{f}\right) \leq J_{f}(X)$. Therefore, for any $\varepsilon>0$, we have

$$
\bigcap_{a \in A_{f}} B\left(a, \frac{J_{f}(X)+\varepsilon}{2}\right) \neq \emptyset .
$$

This implies

$$
\bigcap_{a \in A} B\left(a, \frac{J_{f}(X)+\varepsilon}{2}(R \Phi(X)+\varepsilon)\right) \neq \emptyset .
$$

It follows that $r(A) \leq \frac{J_{f}(X)+\varepsilon}{2}(R \Phi(X)+\varepsilon)$. Since $\varepsilon$ is arbitrary, we obtain $2 r(A) \leq J_{f}(X) \cdot R \Phi(X)$. Therefore $J(X) \leq J_{f}(X) \cdot R \Phi(X)$.

Exactly in the same way, we can prove the following.

Proposition 5.2. We always have $\frac{E(X)}{E_{f}(X)} \leq \Phi(X)$.

An intermediate step, between finite and bcc sets, would be considering compact sets. According to an example given in [31] (see Remark 4.1 in Section 4) it is possible that in $X$ all compact sets, but not all bcc sets, have centers. In the same paper, it is said that it seems to be an open problem whether there are Banach spaces in which all finite sets, but not all compact sets, have centers. We recall the following connected result (see for example [14, Theorem 2.7 (4)]): if for every finite set $A \subset X$ there exists $c$ such that $2 r(A, c)=\delta(A)$, then the same is true for all compact sets.

\section{PRoblems}

In this section, we collect several problems, that we think are open; in some cases we indicate the sections where we have discussed the results suggesting the questions.

6.1 (different sections). Section 2, Proposition 2.1 (3): we know that $J_{f}(X)$ is not always exact. In [21, p.494] an example of a finite dimensional space where $J(X)=J_{f}(X) \neq E(X)$ is given (with a not so clear description). We think that also in infinite dimensional spaces, in general $E_{f}(X) \neq J_{f}(X)$ : give an example (if possible) of such a space where this happens.

Find examples of infinite dimensional spaces where $J(X) \neq E(X)$. Can we characterize the spaces where $E(X)=J(X)$ ? Give an example (if possible) of a space where $E_{f}(X)<E(X)$. Is there any general relation between $J(X)$ and $E_{f}(X)$ ? More precisely: in $c_{0}$ we have $J_{f}(X)=E_{f}(X)<J(X)$; are there spaces where $J(X)<E_{f}(X)$ ? Finally, we remember our Conjecture near the end of Section 3.

6.2 (Sections 2, 4 and 5). Is there any general relation between $J(X)$ and $\Phi(X)$ ? Indeed it is clear that we can have $\Phi(X)<J(X)(\Phi(X)=1$ for any reflexive $X)$. Give an example where $J(X)<\Phi(X)$. Give an example (if possible) where $R \Phi(X)<\Phi(X)$.

6.3 (see Section 2, near the end). Assume that a bcc set $A \subset X$ has no center. Can we always find a set with a center, near to $A$ (in the sense of Hausdorff distance)? If the answer to the first question is not: can we always compute $J(X)$ (or $J_{f}(X)$ ) by only considering bcc sets (resp.: finite sets) with center? 
6.4 (see Section 4, (4.1)). Give "simple" examples showing that, in general, the reverse concerning the second and the third implication in (4.1) do not hold. Can we drop in Proposition 4.2 the assumption concerning existence of centers? Give examples where $(\mathrm{R})$ holds but $\left(\mathrm{R}^{+}\right)$does not.

6.5 (FIP and RFIP). If $X$ satisfies FIP (so $\Phi(X)=1$ ), then $E(X)=E_{f}(X)$ (cf. Proposition 4.3), and moreover (see the discussion before Proposition 4.3) $E(X)$ is exact. We do not know if whenever $X$ satisfies RFIP, $J(X)=J_{f}(X)$ is EXACT (see Proposition 4.3). Also, we do not know if $\Phi(X)=1$ implies FIP (see near the end of Section 4). We think that the RFIP does not imply (GC) (see at the end of Section 4).

6.6 (Propositions 5.1 and 5.2). Does $J(X)=J_{f}(X)$ imply $R \Phi(X)=1$ ? Similar question concerning Proposition 5.2. Otherwise, give examples where we have strict inequality.

\section{Two EXAMPLES AND A SCHEME}

Example 7.1. The following space was introduced in [7, Example 5.2]. Let $c_{0}$ be the usual space of real sequences $\left(x_{n}\right)_{n \in N}$ with the max norm. Set $X=\left\{x \in c_{0}: \sum_{n=1}^{\infty} f_{n} x_{n}=0\right\}$, where the functional $f=\left(f_{1}, \ldots, f_{n}, \ldots\right) \in \ell_{1}$ is the following: $f_{n}=1$ for $n=1,2,3,4 ; f_{n}=\frac{1}{2^{n-4}}$ for $n \geq 5$.

Now consider in $X$ the set $A=\left\{x_{1}, x_{2}, \ldots, x_{n}, \ldots\right\}$, where (for $\left.n \in N\right)$ :

$$
x_{n}=(a, a, a, a, 1, \ldots, 1,0,0,0, \ldots) \text {, }
$$

with: $a=-\frac{1}{4}\left(1-\frac{1}{2^{n}}\right)$; the $5^{\text {th }}, \ldots$, the $(4+n)^{t h}$ component are equal to 1 , and the other components (from the $(5+n)^{t h}$ onwards) are equal to 0 . Clearly $\delta(A)=1$ and $r(A)=1$. This shows that $J(X)=2$. So, it is exact. But the example [7, Example 5.2] shows that there are sets in $X$ without centers: so $J(X)$ is not EXACT.

Apparently, it is not easy to give examples where $J(X) \neq E(X)$; but for single sets it is not difficult to have the corresponding inequality concerning intersections of balls. Next example can illustrate the difference between the IP and the RIP.

Example 7.2. Let $A$ be a set with $\delta(A)=1$ and set $r(A)=r$. The balls $B(a, 1 / 2)(a \in A)$ pairwise intersect; the same is true for the balls $B\left(a, \frac{r(A, a)}{2}\right)$. In fact, given $a_{1}, a_{2}$, a common point for $B\left(a_{1}, \frac{r\left(A, a_{1}\right)}{2}\right)$ and $B\left(a_{2}, \frac{r\left(A, a_{2}\right)}{2}\right)$ is $\frac{a_{1}+a_{2}}{2}$. All the balls $B(a, \lambda / 2)$ with $\lambda>2 r$ intersect (at points which are "almost" centers); also: for $\lambda=2 r$ the intersection, if nonempty, is the set of centers for $A$.

Now consider as $X$ the space $C[-1,1]$. Let $A$ be the set of all functions $f$ such that $0 \leq(x+1) / 2$, if $-1 \leq x \leq 0$ and $0 \leq f(x) \leq 1$, if $0 \leq x \leq 1$. We have $\delta(A)=1, r(A)=1 / 2$. The balls $B(f, \lambda / 2)(f \in A)$ with $\lambda=2 r(A)=1$ intersect: for example, all functions $g \in A: g(x)=1 / 2$ if $0 \leq x \leq 1$, are centers of $A$. But it is not the same for the balls $B\left(f, \lambda \frac{r(A, f)}{2}\right)(f \in A)$ when $\lambda=1$ : indeed it is not difficult to see that their intersection is empty for $\lambda<4 / 3$. In fact, let a function $h$ be in the intersection of all balls $B\left(f, \lambda \frac{r(A, f)}{2}\right) \quad(f \in A)$, for some $\lambda<4 / 3$. Since $A$ contains functions $g$, which are centers of $A$, with value 0 arbitrarily near to 0 , it should satisfy $h \in B(g, \lambda / 4)$. So $h(0)<1 / 3$. But $A$ contains functions $f$ such that $f(x)=1$ for $x=\varepsilon(\varepsilon>0$ and arbitrarily small). Since $r(A, f)=1$ for these functions, we cannot have $\|h-f\| \leq \lambda / 2$ with $\lambda<4 / 3$ for all these functions if $h(0)<1 / 3$.

We summarize in this scheme the implications among different properties. 


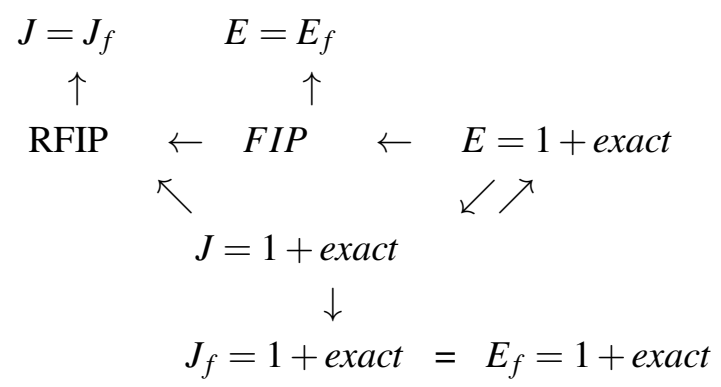

\section{REFERENCES}

[1] A.G. Aksoy, Z. Ibragimov, Convexity of the Urysohn universal space, J. Nonlinear Convex Anal. 17 (2016), 1239-1247.

[2] D. Amir, On Jung's constant and related constants in normed linear spaces, Pacific J. Math. 118 (1985), 1-15.

[3] N. Aronszajn, P. Panitchpakdi, Extension of uniformly transformations and hyperconvex metric spaces, Pacific J. Math. 6 (1956), 405-439.

[4] A. Avilés, F. Cabello Sánchez, J.M.F. Castillo, M. González, Y. Moreno, On Separably Injective Banach Spaces, Lecture Notes in Math. Vol. 2132, Springer, 2016.

[5] P. Bandyopadhyay, S. Dutta, Weighted Chebyshev centres and intersection properties of balls in Banach spaces, Function spaces (Edwardsville, IL, 2002), 43-58; Contemp. Math., 328, Amer. Math. Soc., Providence, RI, 2003.

[6] P. Bandyopadhyay, T.S.S.R.K. Rao, Central subspaces of Banach spaces, J. Approx. Theory 103 (2000), $206-222$.

[7] M. Baronti, E. Casini, P.L. Papini, Equilateral sets and their central points, Rend. Mat. Appl. 13 (1993), 133-148.

[8] M. Baronti, P.L. Papini, Diameters, centers and diametrically maximal sets, in 4th Italian meeting on integral geometry, geometric probability and convex bodies, Bari, Italy (1994), M.I. Stoka ed.; Suppl. Rend. Circ. Mat. Palermo (II), 38 (1995), 11-24.

[9] J.M. Bayod, M.C. Masa, Chebyshev coefficients for $L^{1}$-preduals and for spaces with the extension property, Publ. Mat. 34 (1990), 341-347.

[10] J.M. Bayod, M.C. Masa, Coeficientes de Chebyshev en espacios de funciones continuas, Actas XIV Jornadas HispanoLusas de Matemáticas, La Laguna Vol. I, 233-236, 1990.

[11] J. Benítez, V. Montesinos, On restricted weak upper semicontinuous set valued mappings and reflexivity, Boll. Unione Mat. Ital. (8) 2-B (1999), 577-583.

[12] H.H. Corson, Collections of convex sets which cover a Banach space, Fund. Math. 49 (1960/1961), 143-145.

[13] W.J. Davis, A characterization of $\mathscr{P}_{1}$ spaces, J. Approx. Theory 21 (1977), 315-318.

[14] Y. Duan, B-L. Lin, Characterizations of $L^{1}$-predual spaces by centerable subsets, Comment. Math. Univ. Carolin. 48 (2007), 239-243.

[15] R. Espínola, P. Lorenzo, Metric fixed point theory on hyperconvex spaces: recent progress, Arab. J. Math. 1 (2012), 439-463.

[16] C. Finet, Une classe d'espaces de Banach a prédual unique, Q. J. Math. 35 (1984), 403-414.

[17] C. Franchetti, Relationship between the Jung constant and a certain projection constant in Banach spaces, Ann. Univ. Ferrara 23 (1977), 39-44.

[18] C. Franchetti, Projections onto hyperplanes in Banach spaces, J. Approx. Theory 38 (1983), 319-333.

[19] G. Godefroy, Application á la dualité d'une propriété d'intersection de boules, Math. Z. 182 (1983), 233-236.

[20] G. Godefroy, N. J. Kalton, The ball topology and its applications, Banach space theory (Iowa City, IA, 1987), 195-237, Contemp. Math., 85, Amer. Math. Soc., Providence, RI, 1989.

[21] B. Grünbaum, On some covering and intersection properties in Minkowski spaces, Pacific J. Math. 9 (1959), $487-494$.

[22] B. Grünbaum, Some applications of expansion constants, Pacific J. Math. 10 (1960), 193-201.

[23] M.A. Khamsi, H. Knaust, N.T. Nguyen, M.D. O’Neill, Lambda-hyperconvexity in metric spaces, Nonlinear Anal. 43 (2001), 21-31.

[24] H.E. Lacey, The isometric Theory of Classical Banach Spaces, Springer, Berlin, 1974.

[25] Å. Lima, Intersection properties of balls and subspaces in Banach spaces, Trans. Amer. Math. Soc. 277 (1977), 1-62. 
[26] J. Lindenstrauss, Extension of compact operators, Mem. Amer. Math. Soc. 48 (1964).

[27] P.L. Papini, Completions and balls in Banach spaces, Ann. Funct. Anal. 6 (2015), 24-33.

[28] T.S.S.R.K. Rao, Chebyshev centres and centrable sets, Proc. Amer. Math. Soc. 130 (2002), 2593-2598.

[29] S.O. Schönbeck, On the extension of Lipschitz maps, Ark. Mat. 7 (1967), 201-209.

[30] L. Veselý, Equality of two Banach space constants related to different notions of the radius of a set, Boll. Un. Mat. Ital. 9-A (1995), 515-520

[31] L. Veselý, A Banach space in which all compact sets, but not all bounded sets, admit Chebyshev centers, Arch. Math. 79 (2002), 499-506.

[32] A L. Veselý, Chebyshev centers in hyperplanes of $c_{0}$, Czechoslovak. Math. J. 52 (2002), 721-729. 